\title{
The Current Situation and Future Direction of Traditional and Complementary Medicine (T\&CM) in Malaysian Health Care System Maryam Farooqui*
}

Faculty of Pharmacy, Universiti Teknologi MARA (UiTM), Penang, Malaysia

Malaysia is a multiethnic-multicultural society situated in Southeast Asia. It consists of thirteen states and three federal territories. The total land mass of Malaysia is 329,847 square kilometers which is separated by South China Sea into two regions [1]. The major ethnic groups are Malay (50.4\%), Chinese (23.7\%) and Indians (7\%). Since independence, the country has established a modern health care system, however; T\&CM also built its roots in the health care system. In Malaysia, an estimated US\$ 500 million is spent annually on traditional therapies, compared to only US\$ 300 million on conventional therapies [2]. Malaysian traditional medicines are one of the most valuable and rich cultural heritage of Malaysian people which reflects a diverse culture and tradition. While encouraging and supporting the western health care system in each possible way, the Malaysian cabinet in 2006 approved the proposal to set up an integrated medicine program that incorporates selected T\&CM practices such as Traditional Malay massage and acupuncture for chronic and post stroke management, herbal therapy as an adjuvant treatment for cancer and Malay postnatal care with an aim to ensure the safety and quality of T\&CM for its consumers [3]. Currently, there are a total of ten hospitals with integrated medicine units providing a holistic approach towards enhancing health and quality of life. Both local and foreign traditional practitioners who wish to offer their services in Malaysia are required to register with the T\&CM division under the Ministry of Health, Malaysia. The practitioners are bound to comply with the proposed guidelines in order to offer evidence based practices. The Standard Operating Procedure (SOP) to conduct T\&CM services in the hospital set-up requires patients to be seen by the allopathic practitioners before seeking traditional therapies. However, at present T\&CM treatment is given only as an outpatient.

Ensuring safety, efficacy and quality of T\&CM products is a core objective of World Health Organization (WHO) towards recognizing the role of T\&CM in modern health care system. Research activities in Malaysian T\&CM policy are focused to evidence based medicines. Clinical expertise, research evidence and patient's values and preferences are identified as key components of evidence based practice [4]. An emphasis on evidence based practice is expected to help providers in evaluating clinical effectiveness of treatment while eliminating unsafe practices [5]. To date, several publications such as 'Guidelines on Malay massage and postnatal care, acupuncture, reflexology' and 'Herbal therapies as an adjunct treatment for cancer' have been developed and readily available for both traditional and conventional practitioners who intend to offer T\&CM practices.

Several initiatives have been taken to ensure professional development of traditional practitioners through education and training. Bachelor degree programs in Traditional Chinese Medicines (TCM), Malay medicine, homeopathy, Ayurveda and chiropractic are also offered by local institutes. At the same time, skill based diploma certificate programs namely in Malay massage, acupuncture, Islamic medicine, reflexology and aromatherapy are also offered.

Future advantages of integrating T\&CM in the modern health care system would be reduction of overall health care treatment cost while maintaining the quality of health care. On the other hand, compliance of patients to the conventional health care treatment could be a challenge which could impact health outcomes. Traditional medicines play a complementary role in the national health care system; however concerns have been expressed by conventional medicine practitioners on the need to address the issues related to herbal safety, adulteration, therapeutic approaches, training requirements and professional ethics [6]. Thus integration of T\&CM in the modern health care system requires a great deal of contribution in research from clinical and behavioral aspects. Attitudes of consumers, conventional health care providers, traditional healers as well as policy makers towards integrating T\&CM into the modern health care system may act as a bridge linking research and action to acknowledge the role of T\&CM in supporting human health.

\section{References}

1. http://ms.wikipedia.org/wiki/Malaysia

2. World Health Organization (2002) Traditional Medicine Strategy 2002-2005, Geneva, Switzerland.

3. Editorial Committee, Traditional and Complementary Medicine Division (2011) Traditional and Complementary Medicine Programme in Malaysia.

4. National Policy on Traditional/Complementary Medicine (2002) Malaysian Pharmaceutical Society.

5. Traditional and Complementary medicine program in Malaysia.

6. http://medicine.com.my/wp/category/sections/cam-watch/
${ }^{*}$ Corresponding author: Maryam Farooqui, Universiti Teknologi MARA (UiTM) Penang, Malaysia, E-mail: maryamfarooqui.uitm@gmail.com

Received December 07, 2012; Accepted December 07, 2012; Published December 10, 2012

Citation: Farooqui M (2013) The Current Situation and Future Direction of Traditional and Complementary Medicine ( $T$ and $C M$ ) in Malaysian Health Care System. Altern Integ Med 1:e101. doi:10.4172/2327-5162.1000e101

Copyright: (c) 2013 Farooqui M. This is an open-access article distributed unde the terms of the Creative Commons Attribution License, which permits unrestricted use, distribution, and reproduction in any medium, provided the original author and source are credited. 